INPLASY

PROTOCOL

To cite: Zhang et al.

Effectiveness and safety of floating needle therapy in the treatment of Cervical spondylosis: A protocol for systematic review and metaanalysis. Inplasy protocol 2021120027. doi:

10.37766/inplasy2021.12.0027

Received: 04 December 2021

Published: 04 December 2021

Corresponding author:

Xin Zhang

doctorzhangxin@163.com

Author Affiliation:

Shandong University of

Traditional Chinese Medicine.

Support: No.

SQ2017YFC170007.

Review Stage at time of this submission: Preliminary

searches.

\section{Effectiveness and safety of floating needle therapy in the treatment of Cervical spondylosis: A protocol for systematic review and meta-analysis}

Zhang, ZH1'; Zhang, X2; Tan, QW3; Yu, HJ4; Zhang, CY5; Xian, J6.

Review question / Objective: Is the safety and effectiveness of floating needle therapy treatment for cervical spondylosis better than conventional treatment?

Condition being studied: Cervical spondylosis is a chronic degenerative process of the cervical spine that affects the vertebral bodies and intervertebral disks of the neck, and may progress into disk herniation, bone spur formation, compression of the spinal cord, or cervical spondylotic myelopathy.If it is not a severe acute nerve or spinal cord compression, non-surgical is a good option.Medical treatments include painkillers, antispasmodics, antineuralgics, and muscle relaxants. From acupuncture to massage manipulation to various acupuncture therapies, all play different therapeutic roles in clinical practice.

INPLASY registration number: This protocol was registered with the International Platform of Registered Systematic Review and Meta-Analysis Protocols (INPLASY) on 04 December 2021 and was last updated on 04 December 2021 (registration number INPLASY2021120027).

Conflicts of interest:

None declared.

\section{INTRODUCTION}

Review question / Objective: Is the safety and effectiveness of floating needle therapy treatment for cervical spondylosis better than conventional treatment?

Condition being studied: Cervical spondylosis is a chronic degenerative process of the cervical spine that affects the vertebral bodies and intervertebral disks of the neck, and may progress into disk herniation, bone spur formation, compression of the spinal cord, or cervical spondylotic myelopathy.If it is not a severe acute nerve or spinal cord compression, non-surgical is a good option.Medical treatments include painkillers, 
antispasmodics, antineuralgics, and muscle relaxants. From acupuncture to massage manipulation to various acupuncture therapies, all play different therapeutic roles in clinical practice.

\section{METHODS}

Participant or population: Participants diagnosed with cervical spondylosis will be included with reference to the diagnostic criteria for cervical spondylosis.Diagnostic criteria will include the International Statistical Classification of Diseases and Health Related Problems, 36 Treatment and Rehabilitation of Cervical Spondylosis. There will be no restrictions in terms of age, gender or ethnicity.exclusion criteria: Non-RCT; exclude case reports, cohort studies, duplicate publications, review literature, animal or cellular experiments, and other non-clinical trials.

Intervention: Experimental group. Floating needle therapy includes all combinations of any type of floating needle therapy alone or in combination with other therapies based on floating needles, without limiting the number of treatments or the duration of treatment.

Comparator: The control group will receive one of the following treatments: conventional medication, sham acupuncture (an intervention that mimics "real acupuncture" but does not pierce the skin), no treatment, placebo.

Study designs to be included: Published randomised controlled trials(RCTs with parallel group design, cross-over design) of floating needles for cervical spondylosis in any language will be included.We will not restrict study eligibility by language or publication status.

Eligibility criteria: Participants diagnosed with cervical spondylosis will be included with reference to the diagnostic criteria for cervical spondylosis. Diagnostic criteria will include the International Statistical Classification of Diseases and Health Related Problems,36 Treatment and
Rehabilitation of Cervical Spondylosis. There will be no restrictions in terms of age, gender or ethnicity. Non-RCT; exclude case reports, cohort studies, duplicate publications, review literature, animal or cellular experiments, and other non-clinical trials.

Information sources: In order to obtain the data required for a qualified randomised controlled trials(RCTs), a literature search will be conducted from the following electronic databases: PubMed, Embase, the Cochrane Central Register of Controlled Trials, China National Knowledge Infrastructure(CNKI), Chinese Science and Technology Periodical Database, Wanfang Database, and Chinese Biomedine (CBM)Database.We will search above databases electronically from the beginning to November 2021, without any language restriction.

Main outcome(s): VAS will be considered as the primary outcome indicator.The VAS consists of a $100 \mathrm{~mm}$ straight line, with one end marked 0 for "no pain" and the other end marked 100 for "intolerable pain", A score of $4-6(40-60 \mathrm{~mm})$ is considered moderate pain, and a score of 7-10 $(70-100 \mathrm{~mm})$ is considered severe pain. The total effective rate will be calculated based on the number of cured patients(A1), the number of markedly improved patients(A2), the number of improved patients(A3), and the total patients(A).Total effective rate $=\mathrm{A} 1+\mathrm{A} 2+\mathrm{A} 3 / \mathrm{A}$.

Additional outcome(s): Cervical spine range of motion will be used as the secondary outcome.At the same time, functional impairment level, measured by the Neck Dysfunction Index. Psychological improvement, measured by the Hamilton Anxiety Inventory.

Data management: After the retrieved literature was de-duplicated by the EndNoteX9 software, two researchers read the titles and abstracts of the above literature separately, read the full text of trials that might meet the inclusion criteria to determine if they actually did, and exchanged literature where there were 
contradictions or allowed a third person to decide whether to include them.Two researchers independently extracted study data, including study design, intervention methods, measures, and outcomes, and a third person checked the data for consistency and to ensure the completeness of the required information.We need to ensure that the following information is complete for each article; first author of the article, year of publication, how that exclusion criteria, trial and control groups, treatment duration, sample size, age, gender, outcome indicators, and adverse events.If the above information is incomplete, it is necessary to contact the author.

Quality assessment / Risk of bias analysis: The risk of bias in the study will be determined by two independent investigators using the Cochrane Collaboration tool. The Cochrane Collaboration's tools of the systematic literature review repository include six areas, sequence generation, allocation concealment, blinding, incomplete outcome data, selective reporting and other biases.Areas that need assessment:ls there sufficient random sequence generation (selective bias)? Can allocation be adequately concealed (selective bias)? Was knowledge of the allocation intervention sufficiently blocked during the study period? Were participants and persons of interest (performance bias) outcome assessors (detection bias) and incomplete outcome data adequately addressed (attrition bias) ? Does the study avoid selective outcome reporting (reporting bias)? Is the study sound without other issues that would put it at risk of prejudice?Risk judgments for these terms will be divided into three categories "low risk of bias", "high risk of bias", or "unclear risk of bias".In the event of conflicting differences, risk allocation will be resolved through arbitration by a third party reviewer.

Strategy of data synthesis: Meta-analysis will be performed by RevMan 5.3.0 software. When there was no potential statistical heterogeneity in the results, a fixed-effects model was used for correlation analysis. When there is heterogeneity in the results, the source of heterogeneity will be further analyzed, and after removing the effect of potential heterogeneity, a meta-analysis will be performed using a random-effects model. When clinical heterogeneity was present, we would use subgroup analysis or preliminary analysis of sensitivity, or perform relevant descriptive analysis.

Subgroup analysis: If heterogeneity exists in the meta-analysis, the source of heterogeneity should be sought, such as whether the type of cervical spondylosis, condition, treatment period, duration of treatment with floating needles, and intervention modality are the source of heterogeneity. Therefore, a subgroup analysis should be performed to analyze whether heterogeneity still exists .If a sufficient number of RCTs are included in the review, first, the appropriate subgroup analysis will be performed according to the type of cervical spondylosis (cervical or radicular or vertebral artery) and the intervention results will be analyzed separately.

Sensitivity analysis: Sensitivity analysis is an important method that is mainly used to assess the veracity of the combined data and the accuracy of the meta-analysis results.We progressively remove studies that are of low quality or do not meet the criteria, and then aggregate the data to assess risk bias, measure heterogeneity, sample size, study quality, statistical methods, and the impact of missing data on study results.

\section{Country(ies) involved: China.}

Keywords: floating needle therapy,Cervical spondylosis, protocol, systematic review, meta-analysis.

Contributions of each author:

Author 1 - Zehua Zhang.

Email: doctor20zhangzehua@163.com

Author 2 - Xin Zhang.

Email: doctorzhangxin@163.com

Author 3 - Qiwen Tan. 
Email: tan_qiwen@126.com

Author 4 - Huijuan Yu.

Email: huijuanyu@163.com

Author 5 - Changyun Zhang.

Email: luoriyun1981@163.com

Author 6 - Jin Xian.

Email: jinxian1003@163.com 